\title{
Safety and preliminary efficacy of the Gam-COVID-Vac vaccine and outcomes of SARS-CoV-2 infection in Russian patients with genitourinary malignancies
}

Ilya Tsimafeyeu ${ }^{*}$ (D), Maria Volkova ${ }^{2}$, Galina Alekseeva ${ }^{3}$, Maria Berkut $^{4}$, Alexander Nosov ${ }^{4}$, Igor Myslevtsev ${ }^{5}$, Andrey Andrianov ${ }^{5}$, Andrey Semenov ${ }^{6}$, Pavel Borisov ${ }^{4}$, Ruslan Zukov ${ }^{7}, V_{a d i m}$ Goutnik ${ }^{8}$, Sergey Savchuk ${ }^{9}$, Natalia Dengina ${ }^{10}$ and Timur Mitin ${ }^{11}$

\begin{abstract}
Background: To our knowledge, there is no clinical data pertaining to COVID-19 outcomes and safety of COVID-19 vaccination in Russian patients with genitourinary (GU) malignancies. Aim of our analysis was to describe the characteristics of the COVID-19 infection course as well as preliminary safety and efficacy of Gam-COVID-Vac vaccine in patients with active GU malignancies.

Methods: Patients were retrospectively identified at nine cancer centers in different regions. Patients were included if COVID-19 was diagnosed by a polymerase chain reaction. Data from additional patients with GU cancers who had no positive SARS-CoV-2 RT-PCR test before vaccination and who received two doses of Gam-COVID-Vac (Sputnik V) between 11 February and 31 August 2021 were collected for safety assessment. Anonymized data were collected through an online registry covering demographics, treatments, and outcomes.

Results: The Gam-COVID-Vac vaccine was well tolerated; no grade 3-5 toxicities were reported in 112 vaccinated metastatic GU cancer patients. The most common grade 1 adverse events (81\%) were injection site reactions (76\%), flu-like illness (68\%), and asthenia (49\%). Five patients experienced grade 2 chills (4.5\%) and 3 patients had grade 2 fever (2.7\%). With median follow-up of 6.2 months, two COVID-19 cases were confirmed by RT-PCR test in the vaccine group (of 112 participants; 1.8\%). Eighty-eight patients with COVID-19 disease were included in the analysis. The average age as of the study enrollment was 66 (range 39-81) and the majority of patients were male with renal cell carcinoma (RCC). Thirty-six patients (41\%) had evidence of metastatic disease, of these 22 patients were receiving systemic therapy. More than half of patients required hospitalization. Fifty-four patients (61\%) experienced complications. Sixteen patients who developed COVID-19 pneumonia required mechanical ventilator support. Sixteen patients (18\%) died in a median of 23.5 days after the date of COVID-19 diagnosis was established. The 3-month survival rate was $82 \%$. Clinical and/or radiographic progression of cancer during COVID-19 infection or the subsequent 3 months was observed in 10 patients (11.4\%).
\end{abstract}

*Correspondence: tsimafeyeu@gmail.com

1 Kidney Cancer Research Bureau, Mayakovskogo pereulok 2, Moscow 109147, Russia

Full list of author information is available at the end of the article permits use, sharing, adaptation, distribution and reproduction in any medium or format, as long as you give appropriate credit to the original author(s) and the source, provide a link to the Creative Commons licence, and indicate if changes were made. The images or other third party material in this article are included in the article's Creative Commons licence, unless indicated otherwise in a credit line to the material. If material is not included in the article's Creative Commons licence and your intended use is not permitted by statutory regulation or exceeds the permitted use, you will need to obtain permission directly from the copyright holder. To view a copy of this licence, visit http://creativecommons.org/licenses/by/4.0/. The Creative Commons Public Domain Dedication waiver (http://creativeco mmons.org/publicdomain/zero/1.0/) applies to the data made available in this article, unless otherwise stated in a credit line to the data. 
Conclusion: Patients with GU malignancies are at increased risk of mortality from COVID-19 infection when compared to the general population. Vaccination could be safe in GU cancer patients.

Trial registration: retrospectively registered.

Keywords: COVID-19, Genitourinary malignancies, Mortality, Gam-COVID-Vac vaccine

\section{Introduction}

Despite global measures to combat COVID-19 pandemic, infection rates are on the rise in Russia [1]. Several previously published analyses have revealed a higher risk of death in cancer patients infected with COVID-19. Available to date clinical evidence supports a higher mortality risk from COVID-19 infection in cancer patients in comparison to patients with no active cancer diagnosis. Several meta-analyses have been published since February 2020 [2-4]. These studies used various search and inclusion criteria, but revealed similar statistically significant association between active cancer diagnosis and COVID-19 mortality (relative risk (RR) 1.66-3.16). Severe complications of COVID-19 infection have also been analyzed in patients with or without active cancer diagnosis. The largest meta-analysis to date based on 18 published individual studies revealed a high relative risk of COVID-19 complications in cancer patients (RR 2.48) [5]. Depending on tumor location, presence of metastases, patient performance status and other factors the severity of COVID-19 infection may vary. Patients with lung cancer and mediastinal metastases had the highest morbidity from COVID-19, up to $25-33 \%[5,6]$. To our knowledge, there is no clinical data pertaining to COVID-19 outcomes in patients with genitourinary (GU) malignancies in Russia.

GU cancer patients are at risk for the COVID-19 disease. However, the efficacy and safety profiles of vaccines against SARS-CoV-2 in patients with GU cancers are unknown.

To elucidate the characteristics of COVID-19 infection course, rate of death, and preliminary results of safety and efficacy of the COVID-19 vaccine in patients with active GU malignancies we have performed a retrospective analysis.

We conducted retrospective study to determine the COVID-19 infection course, the mortality rate and safety of the COVID-19 vaccine in patients with active GU malignancies.

\section{Patients and methods}

\section{Vaccinated patients}

We collected data from patients with GU cancers who had no positive SARS-CoV-2 RT-PCR test before vaccination and who were vaccinated between 11 February and 31 August 2021. All patients received two doses of
Gam-COVID-Vac (Sputnik V) intramuscularly with an interval of 21 days. All participants with available data were included in the safety evaluation according to Common Terminology Criteria for Adverse Events (CTCAE) version 5.0. Currently, follow-up is underway for efficacy evaluation.

\section{Unvaccinated patients with COVID-19}

We invited nine comprehensive oncology clinics situated in different geographical regions of Russia to take part in the data collection and analysis. Our study population included all patients with a diagnosis of GU malignancies (prostate cancer, renal cell carcinoma and bladder cancer) who had a positive SARS-CoV-2 RT-PCR test. Symptomatic and asymptomatic COVID-19 patients were included. Asymptomatic patients were diagnosed by RT-PCR test due to established screening procedures in these regions. Age, gender, histology, or stage of disease were not exclusion criteria. Patients receiving active treatment or on active surveillance were included.

Clinical information was extracted from patients' records starting on May 2020 and collected until July 2020. Clinical data were divided into four main categories: (1) demographics, (2) history of cancer diagnosis and management, (3) comorbidities, (4) COVID-19 diagnosis, disease course and outcomes. Oncological outcomes were collected through October 24, 2020 for all patients included in this analysis. Factors selected for the original analysis were selected based on available published data.

All data were de-identified and collected centrally for analysis. The study was conducted according to the criteria set by the Declaration of Helsinki and each subject signed an informed consent before participating to the study.

\section{Statistical analysis}

We carried out descriptive statistics characterizing the disease course of COVID-19 in this population of patients with GU malignancies. Demographic and clinical characteristics were presented as rates and median for continuous variables. In multivariate analysis, we included known factors related to mortality risk, such as age ( $>60$ vs $<60$ years of age), gender, ECOG performance status, presence of metastases, treatment modality (surgery vs systemic therapy vs radiation therapy) and 
cancer type [5]. Patients with missing data were excluded from univariate and multivariate analyses. The proportion was used to calculate the number of adverse events of vaccine and the number of patients infected with SARS-CoV-2.

\section{Results}

\section{Safety and preliminary efficacy of Gam-COVID-Vac} in patients with advanced GU malignancies

The study included 112 vaccinated metastatic GU cancer patients $(59(53 \%)$ patients with renal cell carcinoma, 41 (37\%) patients with prostate cancer, and $12(11 \%)$ patients with bladder cancer; 77 (69\%) patients were male). Median age was 70.6 years (range 29-82). ECOG performance status 0,1 and 2 were determined in 25 (22\%), 71 (64\%) and $16(14 \%)$ patients, respectively. Patients were on active systemic therapy $(44,17,32,18$, and 1 patients received checkpoint inhibitors, tyrosine kinase inhibitors, hormone therapy, chemotherapy, and Radium-223 therapy, respectively). For this interim analysis of safety, data obtained up to September 29, 2021 were analyzed. The most common grade 1 adverse events (81\%) were injection site reactions (76\%), flu-like illness (68\%), and asthenia (49\%). Five patients experienced grade 2 chills $(4.5 \%)$ and three patients had grade 2 fever $(2.7 \%)$. One patient $(0.9 \%)$ had transient ischemic attack as a serious non-treatment related adverse event. Vaccine-related hematologic toxicity was not observed in any patient. The reported adverse events of the vaccine did not lead to interruptions, withdrawal or modification of anticancer treatment. In patients receiving immunotherapy, the vaccine did not affect the risk of immune-mediated adverse events.

With median follow-up of 6.2 months, two COVID19 cases were confirmed by RT-PCR test in the vaccine group (of 112 participants; 1.8\%). One patient received cabazitaxel for advanced prostate cancer and another patient received atezolizumab for metastatic urothelial cancer. In both patients, the course of coronavirus infection was mild and was accompanied by a loss of odors and an increase in temperature up to $38 \mathrm{C}$ during 8 and 15 days, respectively.

\section{COVID-19 disease in unvaccinated patients with GU cancers}

Eighty-eight patients were included in the analysis. All patients had positive SARS-CoV-2 RT-PCR test. Average age as of the study enrollment was 66 (range 39-81) and the majority of patients were male (Table 1 ). The most common GU cancer in this study was renal cell carcinoma, followed by prostate cancer and bladder cancer. The majority of patients were diagnosed with localized or locally advanced cancer. At the time of study, enrollment
36 patients (41\%) had evidence of metastatic disease, of these 22 patients were receiving systemic therapy and for the remaining 14 patients first- or second-line systemic therapy was planned. The most common therapies included checkpoint inhibitors and targeted therapies. In the majority of patients first-line systemic therapy was administered to patients on average 7 days (range $0-11)$ prior to the date of positive SARS-CoV-2 RT-PCR test (Table 1). Three patients elected to continue with prescribed therapy without signs of increased toxicity. Forty-six patients (52\%) underwent surgery no later than 14 days prior to the date of positive SARS-CoV-2 RTPCR test. Fifteen patients (17\%) were receiving radiation therapy at the time of positive SARS-CoV-2 RT-PCR test.

The most common symptoms of COVID-19 infection were fever, fatigue, cough and dyspnea (Table 2). Fifteen patients $(17 \%)$ were asymptomatic. More than half of patients required hospitalization. Median time from onset of symptoms or date of diagnosis and hospitalization was six days (range 2-10). Fifty-four patients (61\%) experienced complications. Pneumonia was the most common complication, and dyspnea was the most common symptom of disease. Sixteen patients who developed COVID-19 pneumonia required mechanical ventilator support (Table 2). Oxygen support was provided to 12 patients (14\%). The remaining patients had mild symptoms and remained isolated at home. ECOG performance status deteriorated during COVID-19 infection in half of study patients.

The median duration of COVID-19 infection was 19.8 days; the median duration of hospitalization was 22.0 days. Sixteen patients $(18 \%)$ died in a median of 23.5 days after the date of COVID-19 diagnosis was established. Three-month survival rate was $82 \%$. In the univariate analysis, advanced age (OR 2.17, 95\% CI 1.18-4.02), renal cell carcinoma (OR 3.72, 95\% CI 2.18.65), presence of distant metastases (OR 6.15, 95\% CI $3.39-10.5$ ), receipt of systemic therapy (OR $2.73,95 \%$ CI 1.11-4.29), ECOG performance status 2 or higher on the date of positive SARS-CoV-2 RT-PCR test (OR 6.1, 95\% CI 3.61-12.07) were factors associated with higher risk of mortality. In the multivariate analysis (Table 3), presence of distant metastases (OR 3.29, 95\% CI 1.77-6.21) and ECOG performance status 2 or higher on the date of positive SARS-CoV-2 RT-PCR test (OR 5.36, 95\% CI 2.84-11.76) remained predictors of higher mortality. The median time of treatment interruption, defined as the interval between the last day of active treatment to the date of return to the same treatment modality, due to COVID-19 diagnosis was 38.9 days (range 18-64).

With median follow-up of 3.5 months, clinical and/or radiographic progression of cancer during COVID-19 infection was observed in 10 patients (11.4\%). Anticancer 
Table 1 Baseline and treatment characteristics of 88 patients

\begin{tabular}{|c|c|}
\hline Number of patients & 88 \\
\hline Age (years), median (range) & $63.5(39-81)$ \\
\hline \multicolumn{2}{|l|}{ Gender } \\
\hline Male & $64(73)$ \\
\hline Female & $24(27)$ \\
\hline \multicolumn{2}{|l|}{ Histology, N (\%) } \\
\hline Renal cell carcinoma & $38(43)$ \\
\hline Prostate cancer & $26(30)$ \\
\hline Urothelial cancer & $24(27)$ \\
\hline \multicolumn{2}{|l|}{ Cancer Stage (UICC, 8th ed.), N (\%) } \\
\hline । & $16(18)$ \\
\hline$\|$ & $16(18)$ \\
\hline III & $20(23)$ \\
\hline IV & $36(41)$ \\
\hline \multicolumn{2}{|l|}{ Distant metastases, N (\%) } \\
\hline No & $52(59)$ \\
\hline Yes: & $36(41)$ \\
\hline Lung & $27(31)$ \\
\hline Liver & $14(16)$ \\
\hline Lymph nodes & $13(15)$ \\
\hline Bone & $10(11)$ \\
\hline Pleura & $5(6)$ \\
\hline Brain & $1(1)$ \\
\hline Pancreas & $1(1)$ \\
\hline \multicolumn{2}{|l|}{ Surgical treatment at the time of COVID-19 } \\
\hline Before surgery, $N(\%)$ & $1(1)$ \\
\hline After surgery, $N(\%)$ & $46(52)$ \\
\hline Time from performed surgery to COVID-19, median (days, range) & $6.5(2-14)$ \\
\hline Radiation therapy at the time of COVID-19, N (\%) & $15(17)$ \\
\hline \multicolumn{2}{|l|}{ Systemic therapy at the time of COVID-19 infection, $N(\%)$} \\
\hline Patients during first-line therapy & $18(21)$ \\
\hline Patients during subsequent therapy & $4(5)$ \\
\hline Checkpoint inhibitors & $8(9)$ \\
\hline Targeted therapy & $7(8)$ \\
\hline Combination of checkpoint inhibitors and & $1(1)$ \\
\hline \multicolumn{2}{|l|}{ Targeted agent } \\
\hline Chemotherapy/gonadotropin releasing hormone analogs & $6(27)$ \\
\hline Not started (in case of initial planning) & $14(16)$ \\
\hline Interrupted (if the patient was on therapy) & $19(9)$ \\
\hline Continued & $3(3)$ \\
\hline
\end{tabular}

treatment was interrupted in all of these patients. Characteristics of these patients are summarized in Table 4. These patients had the diagnosis of renal cell carcinoma $(n=4)$, prostate cancer $(n=4)$ and bladder cancer $(n=2)$. The cancer stage (UICC, 8th edition) distribution among these 10 patients was as follows: Stage I $(n=1)$, Stage III $(n=2)$ and Stage IV $(n=7)$. Among these 10 patients with cancer progression, 6 patients required mechanical ventilator support or oxygen support, 4 patients were admitted to non-intensive care unit in the hospital. All these 10 patients convalesced over a median of 28.8 days.

\section{Discussion}

This is the largest to date retrospective analysis of clinical outcomes among GU cancer patients diagnosed with COVID-19 infection in Russia. Based on our analysis of 88 patients with active GU malignancies, we estimate the mortality risk of $18 \%$ in this group of patients, which is 
Table 2 COVID-19 clinical presentation and outcomes

\begin{tabular}{ll}
\hline Common symptoms of COVID-19, N (\%) & \\
Fever & $60(68)$ \\
Fatigue & $48(53)$ \\
Cough & $39(44)$ \\
Dyspnea & $25(28)$ \\
Complications of COVID-19, N (\%) & \\
Pneumonia & $44(61)$ \\
Respiratory failure & $10(11)$ \\
Multiple organ failure & $8(9)$ \\
Acute respiratory distress syndrome & $6(7)$ \\
Pancreatitis & $2(2)$ \\
Abnormal coagulation & $2(2)$ \\
Anemia & $1(1)$ \\
Frequency of ECOG performance status deterioration during & $48(55)$ \\
COVID-19, $\%$ & \\
Course of COVID-19 & \\
Mild & $46(52)$ \\
Noncritical care hospitalization & $14(16)$ \\
Critical care hospitalization (oxygen support) & $12(14)$ \\
Critical care hospitalization (mechanical ventilation) & $16(18)$ \\
Duration of COVID-19, median (days) & 19.8 \\
Length of hospitalization, median (days) & 22.0 \\
Antiviral/COVID-19 therapy, N (\%) & $42(48)$ \\
Hydroxychloroquine & $31(35)$ \\
Azithromycin & $17(19)$ \\
Dexamethasone & $17(19)$ \\
Favipiravir & $2(2)$ \\
Ritonavir + Lopinavir & $1(1)$ \\
CovID-19 disease outcome & \\
Deanvalescence, N (\%) & \\
\hline
\end{tabular}

similar to the entire population of cancer patients, as previously reported at the annual 2020 ASCO Annual Meeting [7]. In the multivariate analysis, we have identified presence of distant metastases and poor baseline performance status as factors associated with higher COVID19 mortality. We were surprised that age did not remain a predictive factor in our analysis. Subsequent analysis with expansion of patient population will be necessary to determine whether age remains a poor predictor of survival from COVID-19 infection in this group of patients with GU malignancies, as well as clarify the role of active systemic therapy in these patients. Heterogeneity of patients and relatively small sample size are the main limitations of our study.

One-half of patients enrolled on our study experienced mild course of COVID-19 disease, which translated into a relatively high 3 -month survival rate of $82 \%$. The duration of COVID-19 disease -as well as duration of hospitalization-are in line with previously reported numbers [4-7]. At the same time, one-half of patients in our analysis experienced severe symptoms, with pneumonia as the most common clinical presentation. A third of patients with severe COVID-19 infection required oxygen support or mechanical ventilation. The other $16 \%$ were hospitalized for other medical reasons.

Oncological care was interrupted for a period of 18 to 64 days in our study. We cannot exclude that this interruption at least in part contributed to disease progression in $11 \%$ of these patients over the course of subsequent 3 months. At the present time, the safety of observation replacing active management of metastatic GU malignancies during the COVID-19 pandemic remains open and requires additional assessment as well as region-specific policy development. This is particularly relevant for patients with metastatic disease. Nevertheless, 3 patients with GU malignancies infected with COVID-19 elected to continue with their on-going oncological therapies without experiencing adverse outcomes in our study. Thus, with very limited information and no comparison on patients' health conditions between groups with or without anticancer treatment, no solid conclusion should be generated. This limited experience goes against the guidelines and recommendations issued by national and international associations, which advocate for cessation of active anticancer therapies until resolution of COVID19 infection [8-13]. However, COVID-19 vaccination of cancer patients may change the approach to continuing active systemic treatment. Vaccines can be safe and effective [14]. More data are needed on the use of Russian vaccines in GU cancer patients.

Table 3 Multivariable model of factors associated with death

\begin{tabular}{lc}
\hline Factor & Odds ratio $(\mathbf{9 5 \%} \mathbf{C l})$ \\
\hline Age $>60$ years (vs $\leq 60$ years) & $0.79(0.41-1.92)$ \\
Renal cell carcinoma (vs other types) & $1.18(0.55-2.11)$ \\
Distant metastases (vs localized/locally advanced disease) & $3.29(1.77-6.21)$ \\
ECOG performance status $\geq 2$ (vs 0-1) & $5.36(2.84-11.76)$ \\
Systemic therapy (vs surgery vs radiation therapy) & $1.15(0.27-8.90)$ \\
\hline
\end{tabular}


Table 4 Clinical and/or radiographic progression of cancer during COVID-19 disease

\begin{tabular}{ll}
\hline Number of patients & 10 \\
Age (years), mean(range) & $77.4(39-81)$ \\
Gender & \\
Male & $4(40)$ \\
Female & $6(60)$ \\
Cancer Stage (UICC, 8th ed.), N (\%) & \\
I & $1(10)$ \\
II & $0(0)$ \\
III & $2(20)$ \\
IV & $7(70)$ \\
Histology, N (\%) & \\
Renal cell carcinoma & $4(40)$ \\
Prostate cancer & $4(40)$ \\
Urothelial cancer & $2(20)$ \\
Course of COVID-19, N (\%): & \\
Mild & $0(0)$ \\
Mechanical ventilation & $2(20)$ \\
Noncritical care hospitalization & $4(40)$ \\
Critical care hospitalization (oxygen support) & $4(40)$ \\
Duration of COVID-19, median (days) & 28.8 \\
Death as a COVID-19 disease outcome, N (\%) & $4(40)$ \\
\hline
\end{tabular}

\section{Conclusion}

In conclusion, patients with GU malignancies experiencing COVID-19 have higher mortality associated with the presence of metastatic disease and ECOG performance status. The patients who did not interrupt their treatment, did not have excess in complications of COVID-19. Patients who did have an interruption of their treatment, however, might have worse outcome in the long-term follow-up, and we need to be careful to interrupt treatments. Vaccination should be used in cancer patients under the active supervision of an oncologist.

\section{Abbreviations \\ Cl: Confidence interval; CTCAE: Common Terminology Criteria for Adverse Events; ECOG: Eastern Cooperative Oncology Group; GU: Genitourinary; OR: Odds ratio; RR: Relative risk; RT-PCR: Reverse transcription polymerase chain reaction.}

\section{Acknowledgements}

We thank Dr. Maria Mukhina, Dr. Ekaterina Kozlova, and Dr. Kristina Zakurdaeva for their invaluable help.

\section{Authors' contributions}

IT: Project development, data collection, data analysis, manuscript writing. MV: Data collection, data analysis, manuscript editing. GA: Data collection, manuscript editing. MB: Data collection, data analysis, manuscript editing. AN: Data collection, manuscript editing. IM: Data collection, manuscript editing. AA: Data collection, manuscript editing. AS: Data collection, manuscript editing. PB: Data collection, manuscript editing. RZ: Data collection, manuscript editing. VG: Data collection, manuscript editing. SS: Data collection, manuscript editing. ND: Data analysis, manuscript writing. TM: Project development, manuscript writing. All authors read and approved the final manuscript.

\section{Funding}

This study was supported by Kidney Cancer Research Bureau.

\section{Availability of data and materials}

Not applicable.

\section{Declarations}

Ethics approval and consent to participate

All procedures performed in studies involving human participants were in accordance with the ethical standards of the institutional and/or national research committee and with the 1964 Helsinki Declaration and its later amendments or comparable ethical standards.

\section{Informed consent}

Informed consent was obtained from all individual participants included in the study.

\section{Consent for publication}

Not applicable.

\section{Competing interests}

The authors have stated that they have no conflicts of interest.

\section{Author details}

${ }^{1}$ Kidney Cancer Research Bureau, Mayakovskogo pereulok 2, Moscow 109147, Russia. ${ }^{2}$ N.N. Blokhin National Medical Research Center of Oncology, Moscow, Russia. ${ }^{3}$ Pacific State Medical University, Vladivostok, Russia. ${ }^{4}$ N.N. Petrov National Medical Research Center of Oncology, St. Petersburg, Russia. ${ }^{5}$ St. Alexei Central Clinical Hospital, Moscow, Russia. ${ }^{6}$ Ivanovo Regional Oncology Dispensary, Ivanovo, Russia. ${ }^{7}$ V.F. Voyno-Yasenetsky Krasnoyarsk State Medical University, Krasnoyarsk, Russia. ${ }^{8}$ Medicine 24/7 Clinic, Moscow, Russia. ${ }^{9}$ Saint-Petersburg Clinical Scientific and Practical Center for Specialized Types of Medical Care (Oncological), St. Petersburg, Russia. ${ }^{10}$ Ulyanovsk Regional Cancer Center, Ulyanovsk, Russia. ${ }^{11}$ Knight Cancer Institute, Oregon Health and Science University, Portland, OR, USA.

Received: 4 September 2021 Accepted: 26 October 2021

Published online: 13 November 2021

\section{References}

1. Website. Official information on coronavirus in Russia. https://coronavirus. jhu.edu/region/russia. Accsessed 20 Sept 2021.

2. Giannakoulis VG, Papoutsi E, Siempos II. Effect of cancer on clinical outcomes of patients with COVID-19: a meta-analysis of patient data. JCO Glob Oncol. 2020;6:799-808. https://doi.org/10.1200/G0.20.00225.

3. Tian Y, Qiu X, Wang C, et al. Cancer associates with risk and severe events of COVID-19: a systematic review and meta-analysis. Int J Cancer. 2020. https://doi.org/10.1002/ijc.33213.

4. Yang J, Zheng Y, Gou X, et al. Prevalence of comorbidities and its effects in patients infected with SARS-CoV-2: a systematic review and metaanalysis. Int J Infect Dis. 2020;94:91-5. https://doi.org/10.1016/j.jijid.2020. 03.017.

5. Singh AK, Gillies CL, Singh R, et al. Prevalence of co-morbidities and their association with mortality in patients with COVID-19: a systematic review and meta-analysis. Diabetes Obes Metab. 2020. https://doi.org/10.1111/ dom.14124.

6. Garassino MC, Whisenant JG, Huang LC, et al. COVID-19 in patients with thoracic malignancies (TERAVOLT): first results of an international, registry-based, cohort study. Lancet Oncol. 2020;21(7):914-22. https:// doi.org/10.1016/S1470-2045(20)30314-4.

7. Warner JL, Rubinstein S, Grivas P, et al. Clinical impact of COVID-19 on patients with cancer: data from the COVID-19 and cancer consortium (CCC19). J Clin Oncol. 2020;38(18_suppl):Abstract LBA110. https://doi. org/10.1200/JCO.2020.38.18_suppl.LBA110. 
8. Tsimafeyeu I, Volkova M. Management of patients with renal cell carcinoma during coronavirus pandemic: proposed algorithm in Russia. Clin Oncol. 2020;5:1707.

9. Zaorsky NG, Yu JB, McBride SM, et al. Prostate cancer radiation therapy recommendations in response to COVID-19. Adv Radiat Oncol. 2020;5(4):659-65. https://doi.org/10.1016/j.adro.2020.03.010.

10. COVID-19 Resource: European Urology. https://www.europeanurology. com/covid-19-resource. Accessed 04 Apr 2020

11. Ficarra V, Novara G, Abrate A, et al. Research urology network (RUN). Urology practice during the COVID-19 pandemic. Minerva Urol Nefrol. 2020;72(3):369-75.

12. Coronavirus \& COVID-19. The British Association of Urological Surgeons Limited. https://www.baus.org.uk/about/coronavirus_covid-19.aspx. Accessed 03 Apr 2020
13. Tsimafeyeu I, Alekseeva G, Berkut M, et al. COVID-19 in patients with renal cell carcinoma in the Russian Federation. Clin Genitourin Cancer. 2021;19(2):e69-71.

14. Ivanyi P, Grüllich C, Kroeger N, et al. Interdisciplinary working group on renal tumors (IAG-N) of the German Cancer Socity (DKG). Systemic treatment of advanced/metastatic renal cell carcinoma in the contex of SARS-CoV-2 pandemic: recommendations from the interdisciplinary working group for renal tumors (IAG-N). J Cancer Res Clin Oncol. 2020;146(11):3075-8. https://doi.org/10.1007/s00432-020-03341-4.

\section{Publisher's Note}

Springer Nature remains neutral with regard to jurisdictional claims in published maps and institutional affiliations.
Ready to submit your research? Choose BMC and benefit from:

- fast, convenient online submission

- thorough peer review by experienced researchers in your field

- rapid publication on acceptance

- support for research data, including large and complex data types

- gold Open Access which fosters wider collaboration and increased citations

- maximum visibility for your research: over $100 \mathrm{M}$ website views per year

At BMC, research is always in progress.

Learn more biomedcentral.com/submissions 